\title{
Live imaging of newly formed lymphatic vessels in the cornea
}

Cell Research (2011) 21:1745-1749. doi:10.1038/cr.2011.178; published online 15 November 2011

\section{Dear Editor,}

Lymphatic research denotes a field of new discovery and has experienced exponential growth in recent years [1-3]. Though lymphatic dysfunction has been found in a broad spectrum of disorders from transplant rejection to cancer metastasis, to date, there is still little effective treatment for lymphatic diseases, so it is a field with urgent demand for new experimental approaches and therapeutic protocols. The cornea provides an ideal site for lymphatic research due to its accessible location, transparent nature, and alymphatic status under normal condition $[2,4]$. Indeed, the use of this tissue for tumor angiogenesis research dates back to 1970s [5]. Most recently, we have demonstrated that the cornea possesses a full range of plasticity in lymphatic formation and regression [6]. An advanced technology for live imaging of lymphatic vessels in this tissue would therefore have widespread applications in biomedical research.

In this study, we present a novel and highly efficient approach for live imaging of newly formed lymphatic vessels within mouse cornea. Using both normal skin and inflamed cornea, we have demonstrated major features and advantages of this innovative technology. Several examples for applying this new technique to track dynamic lymphatic events in the same tissue over a short or long period of time, or to evaluate the effect of a pharmaceutical intervention during inflammatory lymphangiogenesis, are also presented.

To induce inflammatory lymphangiogenesis and hemangiogenesis in the cornea, we employed the standard suture placement model, as described previously [6-8] Supplementary information, Data S1. Corneal lymphatic vessels were visualized by subconjunctival injection of the large molecular weight fluorescein isothiocyanatelabeled dextran [9]. The dye uptake was continuously monitored under a custom-built live imaging system with an adjustable eye and head holder to secure steady pictures while the mouse is breathing. Two types of live imaging systems were tested: (1) non-contact live imaging system with fluorescent stereomicroscope, and (2) a more advanced live imaging system with two-photon excitation microscope. As demonstrated in Figure 1, while the non-contact system was able to produce high quality images (panels A-D, F, and Supplementary information, Video S1) without touching the cornea, the highly advanced two-photon system yielded more depth-sectioning and cellular details of the tissue and vessels (panel E and Supplementary information, Videos S2, S3 and S4).

This new live imaging technique is sensitive enough to detect lymphatic vessels at various stages, which differ greatly in shapes and degrees of complexity, as illustrated in Figure 1A. A wide range of lymphatic morphologies was visualized, whether they pertained to the early stages of budding and branching, the middle stages of bridging and networking, or the late stage of regressing. The footprints (arrow) of the almost regressed lymphatic vessels were also detectable under higher magnification.

This new live imaging system demonstrated great advantages over conventional ex vivo immunohistochemical assay. One serious limitation to the ex vivo assay is that the method is not applicable for time course evaluation of the same tissue. Previously, to appreciate a glimpse of lymphatic transformation over a certain period of time, a large number of animals have to be sacrificed at different time points for extensive ex vivo assays on dead tissues. The entire process is not only time-, labor-, and resource-demanding, but also fraught with inaccurate information. With the new live imaging system, we were able to follow the same cornea for a short or long period of time to examine dynamic lymphatic changes during the early phase of lymphatic formation, or the late phase of lymphatic regression. As demonstrated in Figure 1B, it was observed that within a short period of $48 \mathrm{~h}$ in the progression phase, an inflammatory lymphatic vessel had developed from a single stem into an elaborated network (top panels). Lymphatic regression, however, occurred more gradually. It took about 2 months for a complex network to become minimal residues, as shown in a long-term 4-month study (middle and lower panels). We anticipate that this great advantage of the new imaging system will facilitate a number of studies in the future to 
A

B
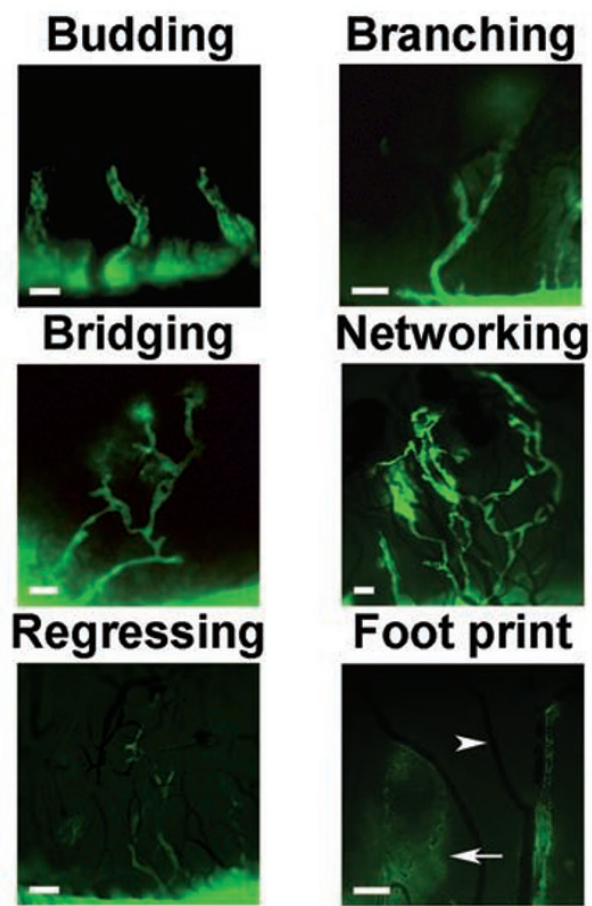

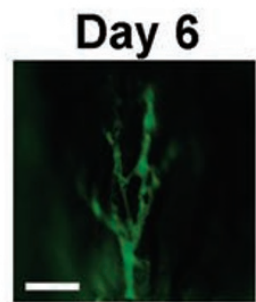

Day 63

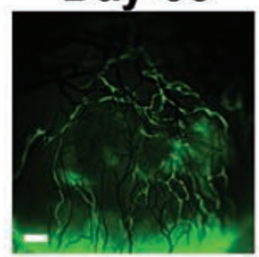

Day 103

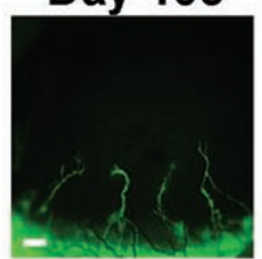

Single light source
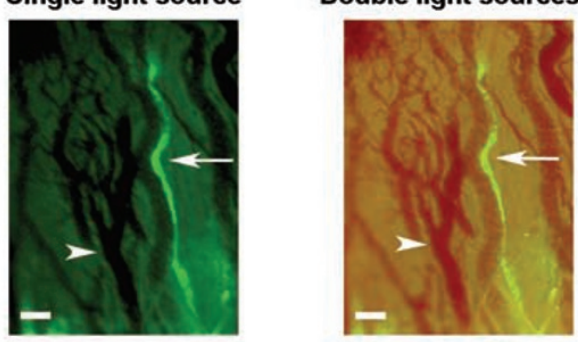

$\mathbf{F}$
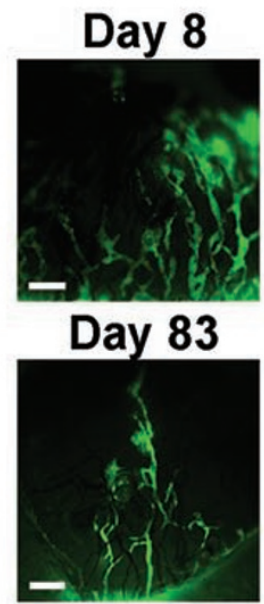

Day 122

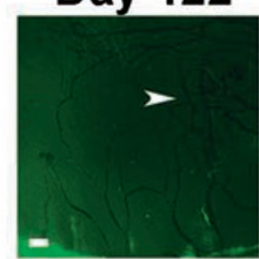

Double light sources

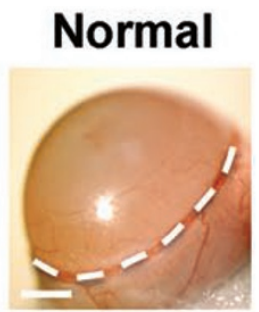

E
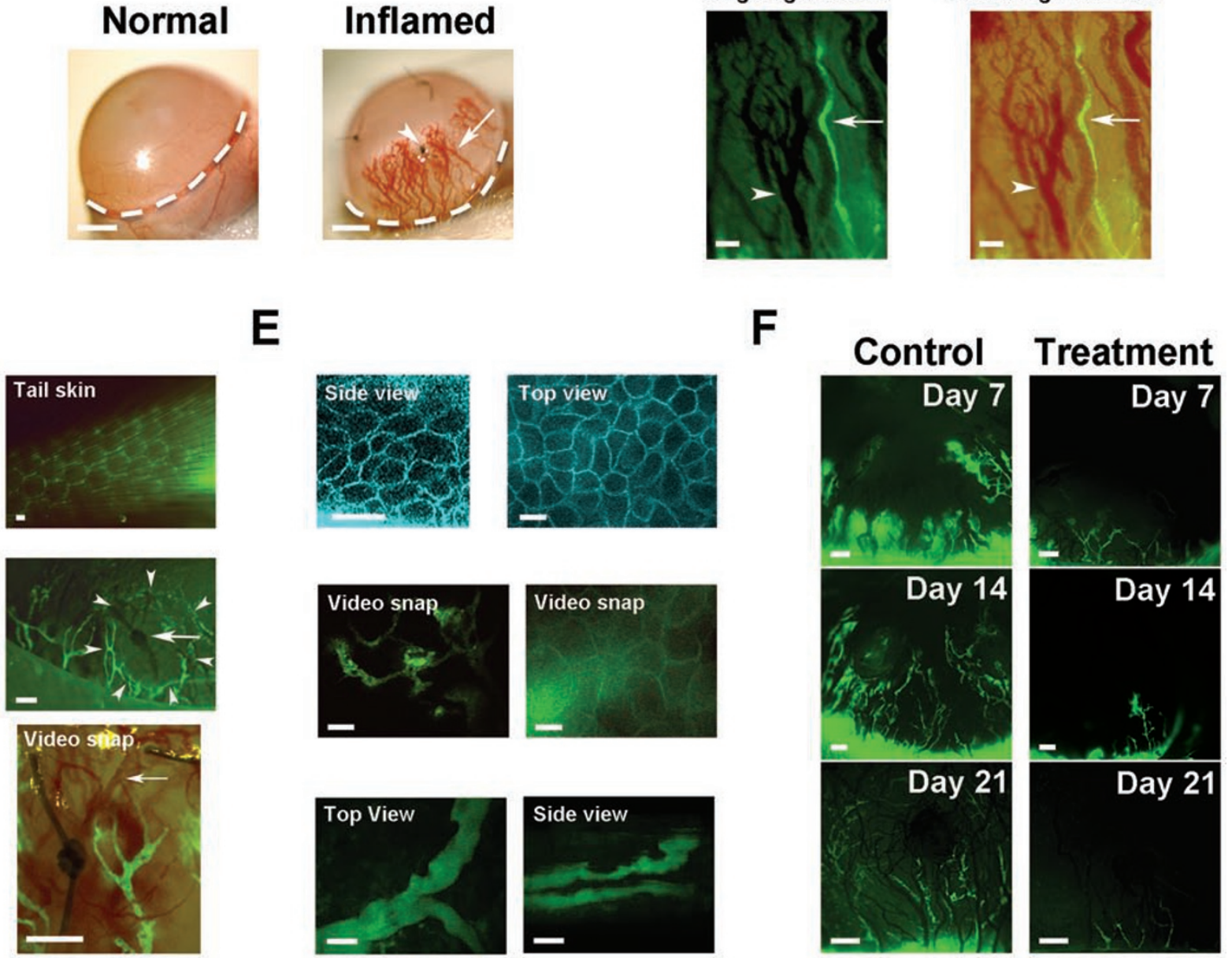
dissect stepwise processes of lymphatic formation, maturation, and regression, or to identify critical time points for therapeutic intervention. We also recognize that the new technique cannot fully substitute for conventional immunohistochemical assays when elucidating the presence or localization of gene expression in lymphatic vessels, or evaluating lymphangiogenesis during embryonic or developmental stages. A combination of both techniques should greatly improve our research capabilities and knowledge in broader lymphatic research fields.

Since blood and lymphatic vessels often accompany each other, and it is important to evaluate both parties in many studies, we next demonstrated the advantages of the new live imaging system over ophthalmic slit-lamp microscopy, which is commonly used in both research laboratories and clinics. As demonstrated in Figure 1C, while only blood vessels (arrow) were detectable by slit-lamp microscopy (left two panels), both blood (arrowheads) and lymphatic (arrows) vessels were clearly revealed by the new system (right two panels). Blood vessels appeared in dark shadows under the fluorescein isothiocyanate fluorescence excitation source alone, whereas they showed up in their natural red color under the combined light sources with a LED bright field. Moreover, the images obtained by the new live imaging system were of better resolution and quality. The fluorescein isothiocyanate-dextran labeled vessels in live corneas expressed LYVE-1, the lymphatic specific marker (data not shown). Since this new technology enables simultaneous observation and tracking of both vessel types, it offers an excellent platform to identify the similarities and disparities between the two vascular systems in response to a stimulus. For example, although not a focus of this study, it was noted that the inflamed lymphatic vessels regressed earlier than blood vessels (arrowheads in Figure 1A and 1B) within live cornea, similarly as reported previously with the immunohistochemistry method [10].

We next demonstrated the advantages of live imaging of lymphatic vessels in the cornea over other nontransparent tissues, such as the skin, which is normally endowed with lymphatic vessels. As shown in Figure 1D, live imaging of tail skin, a site used for lymphatic imaging in previous studies, revealed a cross-sectional view of the lymphatics, which was obscured by a layer of body hairs (top panel). In contrast, imaging within the cornea, a transparent tissue free of any preexisting or background vessels, showed an entire tree of the newly formed lymphatic vessels from their peripheral roots to central branches (middle panel). This method also enables observation of lymphatic vessels in the context of their local and physiological environment, which harbored newly formed blood vessels and the site of pathological stimulation as well. We here observed an interesting phenomenon showing terminal lymphatics (arrowheads) encircling a suture spot (arrow). More dynamic biological events, such as rapid red blood cell circulation (arrow) within blood vessels, were also detected and recorded in time-lapse movies (lower panel and Supplementary information, Video S1).

To obtain more details of the cornea at a cellular level, we next performed live imaging with a custom-built and highly advanced two-photon excitation fluorescence microscope. The system reaches a $z$-resolution of $0.5 \mu \mathrm{m}$ with the high numerical aperture objective and allows for a precise evaluation of the most delicate features of the lymphatic vessels within live corneas. As illustrated in Figure 1E, detailed structures of the cornea were revealed showing well-organized epithelial cells in top layers (top panels) and newly formed lymphatic vessels in the

Figure 1 Live imaging of newly formed lymphatic vessels in inflamed cornea. (A) Lymphatic vessels of various shapes and complexity. Arrow: footprints of an almost regressed lymphatic vessel; Arrowhead: blood vessel. (B) Longitudinal tracking of dynamic lymphatic processes in the same cornea in the progression (top panels) or regression phase (middle and lower panels). Arrowhead: blood vessel. (C) Comparison between ophthalmic slit-lamp microscopy (left two panels; scale bars: 1000 $\mu \mathrm{m}$ ) and the new imaging system, which also reveals lymphatic vessels (right two panels) in the corneas 2 weeks after suture placement. Right two panels: Arrows, lymphatic vessels; Arrowheads, blood vessels. (D) Comparison between live imaging of lymphatic vessels in normal tail skin and inflamed cornea 2 weeks after suture placement. Top: cross-sectional view of background lymphatics in the skin. Middle: entire morphological tree of new lymphatic vessels in the cornea showing terminal lymphatics (arrowheads) encircling a suture spot (arrow). Lower: snap picture (Supplementary information, Video S1) showing rapid red blood cell flow (arrow) inside new blood vessels. (E) Advanced two-photon live images showing ultra-structure of the cornea with lymphatic vessels. Top: side and top views of epithelial cells in layers. Middle: snap pictures showing lymphatic vessels in the stroma. Left (Supplementary information, Video S2): low magnification view taken along the central-toperipheral axis. Right (Supplementary information, Video S3): high magnification view taken along the superficial-to-deep axis with lymphatic vessels behind the epithelium. Lower: snap pictures (Supplementary information, Video S4) showing two lymphatic vessels that appeared to cross over each other from the top view (left) were located at different layers from a side view (right). Pictures are assigned pseudo colors. Scale bars: $20 \mu \mathrm{m}$. (F) Real time in vivo evaluation of the effect of systemic VEGFR-2 blockade on corneal inflammatory lymphangiogenesis. Scale bars: $100 \mu \mathrm{m}$ unless otherwise indicated. 
stroma (middle and lower panels). This delicate system enabled precise positioning of lymphatic vessels in close proximity. As shown in the lower panels, it was found that two lymphatic vessels that appeared to cross over each other from the top view (left) were actually located at different layers from a side view (right). We hope this great capacity will prove powerful in future studies to examine lymphatic vessels at close proximity, or to quantify lymphatic branching points. The ultrastructural information was recordable in multidimensional timelapse movies, which could be taken under low-to-high magnifications and along various axes (Supplementary information, Videos S2, S3 and S4).

Finally, to demonstrate an application of this new technology in the evaluation of a pharmacological intervention in inflammatory lymphangiogenesis, we performed a longitudinal study on the consequences of systemic administrations of vascular endothelial growth factor receptor-2 neutralizing antibodies on inflammatory lymphangiogenesis within live corneas. As shown in Figure $1 \mathrm{~F}$, a brief treatment regimen with two injections at day 0 and day 3 after the suture placement had led to a significant reduction of lymphatic growth in both density and length. These data also provide the first in vivo imaging evidence showing that vascular endothelial growth factor receptor-2 is critically involved in inflammatory lymphangiogenesis in the cornea.

In summary, we here describe a novel and innovative technique that can be used for multidimensional imaging of newly formed lymphatic vessels in live mouse cornea. Our imaging system generates high quality pictures showing lymphatic vessels in clear contrast against the background. To our knowledge, this is the first report on direct visualization of lymphatic vessels within live mouse cornea, one of the most desirable sites for vascular research. Since there are no preexisting or background vessels to consider, it is exceptionally straightforward and accurate to assess new lymphatic events at this site, regardless of whether they are related to a pathological stimulation or a pharmaceutical intervention, as demonstrated in this study.

Yet to be demonstrated, it is possible to apply this new technique to investigate the interplay between lymphatic vessels with other cellular, molecular, or nerve components in the environments. For example, mouse strains with specific fluorescent reporters for dendritic cells, macrophages, or nerve fibers may be used in combination with this new technology to advance our research goals in the related fields. It is also possible to employ this new technology to examine lymphatic processes induced by other biological or pathological insults, such as growth factors, infections, tumors, trauma, transplan- tation, or chemical burn, of which various models have been developed in mouse cornea [4].

Taken together, we anticipate that this new live imaging technology will facilitate diverse applications in broad biomedical fields. Given its high efficiency and accuracy, we hope this new technique will become a powerful tool for anyone studying lymphatic vessels, inside or outside the eye.

Disclosure: Dr Bronek Pytowski is employed by ImClone Systems, a wholly owned subsidiary of Eli Lilly and Company. The company is developing antagonist antibodies to vascular endothelial growth factor receptor-2 for the treatment of cancer.

\section{Acknowledgments}

This work is supported in part by research grants from the National Institutes of Health and the University of California at Berkeley (LC). XW received scholarship from Shanghai Jiao Tong University. We acknowledge Paul Herzmark and Ellen Robey at the Center for Host-Pathogen Studies Imaging Core and Yang Dan at Department of Molecular and Cell Biology (University of California, Berkeley, USA) for assistance with microscopy.

\section{Don Yuen ${ }^{1, *}$, Xiufeng $\mathrm{Wu}^{1,2,{ }^{1}}$, Alex C Kwan ${ }^{3}$, Jeffrey LeDue ${ }^{1}$, Hui Zhang ${ }^{1}$, Tatiana Ecoiffier ${ }^{1}$, Bronislaw Pytowski ${ }^{4}$, Lu Chen ${ }^{1,5,6}$}

${ }^{I}$ Center for Eye Disease and Development, Program in Vision Science, University of California, Berkeley, CA 94720, USA; ${ }^{2}$ School of Medicine, Shanghai Jiao Tong University, China; ${ }^{3}$ Department of Molecular and Cell Biology, Helen Wills Neuroscience Institute, University of California, Berkeley, USA, ${ }^{4}$ Cell Biology and Bioimaging, ImClone Systems, Eli Lilly and Company, New York, USA; ${ }^{5}$ The Francis I. Proctor Foundation for Research in Ophthalmology, University of California, San Francisco, USA; ${ }^{6}$ Schepens Eye Research Institute, Harvard Medical School, Boston, USA

*These two authors contributed equally to this work. Correspondence: Lu Chen

E-mail: chenlu@berkeley.edu

\section{References}

1 Tammela T, Alitalo K. Lymphangiogenesis: Molecular mechanisms and future promise. Cell 2010; 140:460-476.

2 Chen L. Ocular lymphatics: state-of-the-art review. Lymphology 2009; 42:66-76.

3 Oliver G, Detmar M. The rediscovery of the lymphatic system: old and new insights into the development and biological function of the lymphatic vasculature. Genes Dev 2002; 16:773783.

4 Chen L, Hann B, Wu L. Experimental models to study lymphatic and blood vascular metastasis. J Surg Oncol 2011; 103:475-483. 
5 Gimbrone MA Jr, Cotran RS, Leapman SB, Folkman J. Tumor growth and neovascularization: an experimental model using the rabbit cornea. J Natl Cancer Inst 1974; 52:413-427.

6 Zhang H, Hu X, Tse J, Tilahun F, Qiu M, Chen L. Spontaneous lymphatic vessel formation and regression in the murine cornea. Invest Ophthalmol Vis Sci 2011; 52:334-338.

7 Chen L, Hamrah P, Cursiefen C, et al. Vascular endothelial growth factor receptor-3 mediates induction of corneal alloimmunity. Nat Med 2004; 10:813-815.
8 Grimaldo S, Yuen D, Ecoiffier T, Chen L. Very late antigen-1 mediates corneal lymphangiogenesis. Invest Ophthalmol Vis Sci 2011; 52:4808-4812.

9 Swartz MA, Berk DA, Jain RK. Transport in lymphatic capillaries. I. Macroscopic measurements using residence time distribution theory. Am J Physiol 1996; 270:H324-H329.

10 Cursiefen C, Maruyama K, Jackson DG, Streilein JW, Kruse FE. Time course of angiogenesis and lymphangiogenesis after brief corneal inflammation. Cornea 2006; 25:443-447.

(Supplementary information is linked to the online version of the paper on the Cell Research website.) 